Kragujevac Journal of Mathematics

Volume 42(3) (2018), Pages 431-439.

\title{
CONVERGENCE ANALYSIS OF LEAST SQUARES-EPSILON-RITZ ALGORITHM FOR SOLVING A GENERAL CLASS OF PANTOGRAPH EQUATIONS
}

\author{
S. A. YOUSEFI ${ }^{1}$, M. NOEI KHORSHIDI ${ }^{1}$, AND A. LOTFI $^{1}$
}

\begin{abstract}
In this paper, we propose an approximate method for solving a general class of pantograph differential equations. The proposed method is based on a combination of least squares, epsilon and Ritz methods. The convergence properties of the method are analyzed and discussed. Finally, several numerical examples are given to illustrate the applicability and efficiency of the method.
\end{abstract}

\section{INTRODUCTION}

Pantograph equations are a kind of functional differential equations which have wide applications in various fields such as analytic number theory, nonlinear dynamical systems and probability theory on algebraic structures $[3,13,14]$. The name pantograph originated from the Ockendon and Tayler's work on the collection of current by the pantograph head of an electric locomotive [16]. In recent years there has been the growing interest in the pantograph equations $[1,3,11,15]$. Pantograph equations are usually difficult to solve analytically, so numerical methods are required. There has been number of some numerical methods for solving pantograph equations are presented by researchers of this field. So far various numerical methods have been proposed to solve pantograph equations such as the variational and modified variational iteration method $[2,11,19,27]$, the homotopy method [28], Taylor polynomial method [21-23], the Bessel collocation method [24,29,30], the reproducing kernel space method [4], the discontinuous Galerkin methods [1], the Runge-Kutta methods [9] and [10], the $\theta$-methods [25] and [26], the Chebyshev polynomials method [20], the Hermite

Key words and phrases. Epsilon method, Least squares method, Pantograph equations, Ritz method.

2010 Mathematics Subject Classification. Primary: 65N30. Secondary: 35A24.

Received: May 05, 2016.

Accepted: April 15, 2017. 
method [31], the rational approximation method [7], the linear multistep methods [6], the shifted Legendre approximation method [32], the generalized fractional-order Bernoulli wavelet method [17] and the shifted orthonormal Bernstein polynomials method [8].

In this work, by combining least square and epsilon methods $[5,12]$ we present an efficient method for solving the multi-pantograph equation. The remainder of the paper is structured as follows. After explaining the problem, we obtain the least squares-Epsilon method for solving the multi-pantograph equation in Section 3 and Section 4. In Section 5, the convergence results of the least squares-epsilon method are given. We use numerical example to confirm the efficiently of the method in Section 6.

\section{Statement of the Problem}

Our goal in this article is to find approximate solution for multi-pantograph equation of the following form

$$
\begin{aligned}
& \sum_{i=1}^{\rho} \frac{f_{i}(x)}{x^{l}(x-\lambda)^{d}} u^{i}(x)+\sum_{i=1}^{\bar{\rho}} \frac{g_{i}(x)}{x^{l}(x-\lambda)^{d}} u^{(i)}(x)+\sum_{i=1}^{\delta} \sum_{j=1}^{\gamma} \frac{\alpha_{i, j}(x)}{x^{l}(x-\lambda)^{d}} u^{i}\left(p_{j} x\right) \\
+ & \sum_{i=1}^{\bar{\delta}} \sum_{j=1}^{\bar{\gamma}} \frac{\beta_{i, j}(x)}{x^{l}(x-\lambda)^{d}} u^{(i)}\left(q_{j} x\right)=h(x), \quad 0<x<\lambda,
\end{aligned}
$$

with boundary conditions

$$
u^{(i)}(0)=\eta_{0, i}, \quad u^{(j)}(\lambda)=\eta_{1, j}
$$

$i=0,1, \ldots, n_{1}-1, j=0,1, \ldots, n_{2}-1, n_{1}+n_{2}=n=\max \{\bar{\rho}, \bar{\delta}\}$, where $0<p_{i}, q_{j}<1$ and $l, d \in \mathbb{Z}^{+}$. Here we suppose that $f_{i}(x), g_{i}(x), \alpha_{i, j}(x), \beta_{i, j}(x), h(x) \in C(0, \lambda)$. In this problem unknown function $u(x)$ belongs to the space $C^{n}(0, \lambda)$.

The problem (2.1) with boundary conditions (2.2) is a singular equation defined on the open interval $(0, \lambda)$. In the subsequent development, we proceed solving the following problem

$$
\begin{gathered}
\sum_{i=1}^{\rho} f_{i}(x) u^{i}(x)+\sum_{i=1}^{\bar{\rho}} g_{i}(x) u^{(i)}(x)+\sum_{i=1}^{\delta} \sum_{j=1}^{\gamma} \alpha_{i, j}(x) u^{i}\left(p_{j} x\right) \\
+\sum_{i=1}^{\bar{\delta}} \sum_{j=1}^{\bar{\gamma}} \beta_{i, j}(x) u^{(i)}\left(q_{j} x\right)=x^{l}(x-\lambda)^{d} h(x), \quad 0 \leqslant x \leqslant \lambda, \\
u^{(i)}(0)=\eta_{0, i}, \quad u^{(j)}(\lambda)=\eta_{1, j},
\end{gathered}
$$

$i=0,1, \ldots, n_{1}-1, j=0,1, \ldots, n_{2}-1, n_{1}+n_{2}=n=\max \{\bar{\rho}, \bar{\delta}\}$, where $u(x) \in C^{n}[0, \lambda]$ and $f_{i}(x), g_{i}(x), \alpha_{i, j}(x), \beta_{i, j}(x), h(x) \in C[0, \lambda]$. It is obvious that the solution of the problem (2.3) with conditions (2.4) also satisfies the problem (2.1) with conditions $(2.2)$. 


\section{LEAST SQUARES-EPSILON METHOD}

In this section, we develop an approximate method for solving problem (2.3) with conditions (2.4) by combining least square and epsilon methods. Without loss of generality, we consider $\lambda=1$ in (2.3) and (2.4). Consider Equation (2.3) in the following form

$$
\begin{aligned}
& F\left(x, u(x), u^{(1)}(x), \ldots, u^{(\bar{\rho})}(x), u\left(p_{1} x\right), \ldots, u\left(p_{\gamma} x\right), u^{(1)}\left(q_{1} x\right), \ldots, u^{(1)}\left(q_{\bar{\gamma}} x\right), \ldots,\right. \\
& \left.u^{(\bar{\delta})}\left(q_{1} x\right), \ldots, u^{(\bar{\delta})}\left(q_{\bar{\gamma}} x\right)\right)=0, \quad 0 \leq x \leq 1 .
\end{aligned}
$$

Define functional $J: M \rightarrow \mathbb{R}$ as follows

$$
J[u]:=\int_{0}^{1} F^{2} d x=\|F\|_{L^{2}}^{2},
$$

where

$$
\begin{aligned}
M:= & \left\{u \in C^{n}[0,1]: u^{(i)}(0)=\eta_{0, i}, u^{(j)}(1)=\eta_{1, j}, i=0,1, \ldots, n_{1}-1,\right. \\
& \left.j=0,1, \ldots, n_{2}-1\right\} .
\end{aligned}
$$

Now we assume that Equation (2.3) has a solution $u_{e x}$ in $M$. So, the functional $J$ has a minimizing solution $u_{e x} \in M$ with minimum value $J\left[u_{e x}\right]=0$. Our purpose is to find an approximate minimizing solution for the functional $J$ given by (3.2) on $M$. The minimization problem min $\left.J\right|_{M}$ is a constrained optimization problem. By applying the epsilon method, we transform the constrained optimization problem into the following unconstrained problem

$$
\tilde{J}[u]=\int_{0}^{1} F^{2} d x+\frac{1}{\epsilon}\left(\sum_{i=0}^{n_{1}-1}\left(u^{(i)}(0)-\eta_{0, i}\right)^{2}+\sum_{j=0}^{n_{2}-1}\left(u^{(j)}(1)-\eta_{1, j}\right)^{2}\right),
$$

where $\epsilon>0$ is given.

We minimize the functional $\tilde{J}$ on $C^{n}[0,1]$. It is obvious that $u_{e x}$ is also a minimizing solution for $\tilde{J}$ for any considered value of $\epsilon$ and $\tilde{J}\left[u_{e x}\right]=0$. Theorem 5.2 ensures that solving the problem $\left.\min \tilde{J}\right|_{C^{n}[0,1]}$ by utilizing the Ritz method leads to an approximate minimizing solution for the functional $J$ on $M$.

\section{Approximate solution of PANtograph EQUation}

Consider expansion $u_{k, \epsilon}(t)$, in the following form

$$
u_{k}(t)=C_{k}^{T} \cdot \Psi_{k}(t), \quad \Psi_{k}(t)=\left(\begin{array}{c}
\phi_{0}(t) \\
\phi_{1}(t) \\
\vdots \\
\phi_{k}(t)
\end{array}\right), \quad C_{k}=\left(\begin{array}{c}
c_{0} \\
c_{1} \\
\vdots \\
c_{k}
\end{array}\right)
$$

Here, $\phi_{j}(t), j \in\{0\} \cup \mathbb{N}$ are shifted Legendre orthonormal polynomials

$$
\phi_{j}(t)=\sqrt{2 j+1} \sum_{k=0}^{j}(-1)^{j+k} \frac{(j+k) ! t^{k}}{(j-k) !(k !)^{2}}, \quad j=0,1,2, \ldots, t \in[0,1] .
$$


Substituting $u_{k}$ into (3.5) gives us

$$
\tilde{J}\left[c_{0}, \ldots, c_{k}\right]=\int_{0}^{1} F_{k}^{2} d x+\frac{1}{\epsilon}\left(\sum_{i=0}^{n_{1}-1}\left(u_{k}^{(i)}(0)-\eta_{0, i}\right)^{2}+\sum_{j=0}^{n_{2}-1}\left(u_{k}^{(j)}(1)-\eta_{1, j}\right)^{2}\right),
$$

where

$$
\begin{aligned}
F_{k}:= & F\left(x, u_{k}(x), u_{k}^{(1)}(x), \ldots, u_{k}^{(\bar{\rho})}(x), u_{k}\left(p_{1} x\right), \ldots, u_{k}\left(p_{\gamma} x\right), u_{k}^{(1)}\left(q_{1} x\right), \ldots,\right. \\
& \left.u_{k}^{(1)}\left(q_{\bar{\gamma}} x\right), \ldots, u_{k}^{(\bar{\delta})}\left(q_{1} x\right), \ldots, u_{k}^{(\bar{\delta})}\left(q_{\bar{\gamma}} x\right)\right),
\end{aligned}
$$

which is an algebraic function of unknowns $c_{j}, j=0,1, \ldots, k$. If $c_{j}$ s be determined by minimizing function $\tilde{J}$, then by (4.1) we achieve function that approximate minimum value of $\tilde{J}$ in (4.3). According to differential calculus, the following system of equations is the necessary condition of optimization for the multidimensional function (4.3)

$$
\frac{\partial \tilde{J}}{\partial c_{j}}=0, \quad 0 \leq j \leq k .
$$

By solving system (4.4), we can determine minimizing values of $c_{j}, j=0,1, \ldots, k$, for function (4.3). Hence, we achieve functions $u_{j}$, by (4.1), which approximate minimum value of $J$ by

$$
J\left[c_{0}, \ldots, c_{k}\right]=\int_{0}^{1} F_{k}^{2} d x
$$

while

$$
\begin{aligned}
& u_{k}^{(i)}(0) \simeq \eta_{0 . i}, \quad i=0,1, \ldots, n_{1}-1, \\
& u_{k}^{(j)}(1) \simeq \eta_{1, j}, \quad j=0,1, \ldots, n_{2}-1 .
\end{aligned}
$$

\section{ON THE CONVERGENCE OF THE METHOD}

In this section we discuss the convergence of the method presented in Section 4. Lemma 5.1 shows that the functional $\tilde{J}$ is continuous on $C^{n}[0,1]$ with respect to the norm, $\|\cdot\|_{n}$, defined as follows

$$
\|u\|_{n}=\|u\|_{\infty}+\cdots+\left\|u^{(n)}\right\|_{\infty} .
$$

We use this important property later in Theorem 5.2. The following theorem from real analysis plays key roll in the proof of Lemma 5.1.

Theorem 5.1. Let $f$ be continuous mapping of a compact metric space $X$ into a metric space $Y$, then $f$ is uniformly continuous.

Proof. See [18].

Lemma 5.1. The functional $J$ is continuous on $C^{n}[0,1]$. 
Proof. Let $u^{*} \in C^{n}[0,1]$. Notice that $\Delta>0$ is given. Consider $d>0$ and

$$
I=[0,1] \times \prod_{i=1}^{1+\bar{\rho}+\gamma+\bar{\gamma} \bar{\delta}}[-L-d, L+d],
$$

where $L=\max \left\{\|u\|_{\infty},\left\|u^{\prime}\right\|_{\infty}, \ldots,\left\|u^{(n)}\right\|_{\infty}\right\}$. Obviously

$$
\begin{aligned}
U^{*}(x):= & \left(x, u^{*}(x), u^{*(1)}(x), \ldots, u^{*(\bar{\rho})}(x), u^{*}\left(p_{1} x\right), \ldots, u^{*}\left(p_{\gamma} x\right), u^{*(1)}\left(q_{1} x\right), \ldots,\right. \\
& \left.u^{*(1)}\left(q_{\bar{\gamma}} x\right), \ldots, u^{*(\bar{\delta})}\left(q_{1} x\right), \ldots, u^{*(\bar{\delta})}\left(q_{\bar{\gamma}} x\right)\right) \in I, \quad x \in[0,1] .
\end{aligned}
$$

$\Delta_{1}>0$ is given. Let $\delta_{1}>0$ and $\left\|u-u^{*}\right\|_{n}<\delta_{1}$, then we have

$$
\begin{gathered}
\left\|u^{(j)}-u^{*(j)}\right\|_{\infty}<\delta_{1}, \quad 1 \leq j \leq \bar{\rho}, \\
\max \left\{\left|u\left(p_{j} x\right)-u^{*}\left(p_{j} x\right)\right|: x \in[0,1]\right\}<\delta_{1}, \quad 1 \leq j \leq \gamma, \\
\max \left\{\left|u^{(i)}\left(q_{j} x\right)-u^{*(i)}\left(q_{j} x\right)\right|: x \in[0,1]\right\}<\delta_{1}, \quad 1 \leq j \leq \bar{\gamma}, 1 \leq i \leq \bar{\delta} .
\end{gathered}
$$

According to (5.2)-(5.4) it is easy to see that for small enough value of $\delta_{1}$ we have

$$
\begin{gathered}
U(x):=\left(x, u(x), u^{(1)}(x), \ldots, u^{(\bar{\rho})}(x), u\left(p_{1} x\right), \ldots, u\left(p_{\gamma} x\right), u^{(1)}\left(q_{1} x\right), \ldots, u^{(1)}\left(q_{\bar{\gamma}} x\right), \ldots,\right. \\
\left.u^{(\bar{\delta})}\left(q_{1} x\right), \ldots, u^{(\bar{\delta})}\left(q_{\bar{\gamma}} x\right)\right) \in I, \quad x \in[0,1], \\
\left|U(x)-U^{*}(x)\right|<\Delta_{1}, \quad x \in[0,1] .
\end{gathered}
$$

Since function $F$ is continuous on $I$ and $I$ is a compact set, according to Theorem $5.1, F$ is uniformly continuous on $I$. So if $\delta_{1}>0$ be sufficiently small, then $\left|U(x)-U^{*}(x)\right|<$ $\Delta_{1}$ implies that $\left|F(U(x))-F\left(U^{*}(x)\right)\right|<\Delta, x \in[0,1]$, and $\left|J[u]-J\left[u^{*}\right]\right|<\Delta$.

Define $L_{\epsilon}: C^{n}[0,1] \rightarrow \mathbb{R}$ as follows

$$
L_{\epsilon}[u]:=\frac{1}{\epsilon}\left(\sum_{i=0}^{n_{1}-1}\left(u^{(i)}(0)-\eta_{0, i}\right)^{2}+\sum_{j=0}^{n_{2}-1}\left(u^{(j)}(1)-\eta_{1, j}\right)^{2}\right) .
$$

It is easy to observe that the functional $L_{\epsilon}$ is continuous on $C^{n}[0,1]$ with respect to $\|\cdot\|_{n}$. So by Lemma 5.1 , for any selection of $\epsilon \in \mathbb{R}$, the functional $\tilde{J}$ is continuous on $C^{n}[0,1]$.

Theorem 5.2. Let $\hat{\mu}_{k}=\tilde{J}\left[u_{k}\right]$ be the minimum of the functional $\tilde{J}$ on $P_{k}[0,1]$ and $\mu_{k}:=J\left[u_{k}\right]$, then

$$
\lim _{k \rightarrow \infty} \mu_{k}=0
$$

and

$$
\begin{aligned}
& \lim _{j \rightarrow \infty}\left|u_{k}^{(i)}(0)-\eta_{0 . i}\right|=0, \quad i=0,1, \ldots, n_{1}-1, \\
& \lim _{j \rightarrow \infty}\left|u_{k}^{(i)}(1)-\eta_{1, i}\right|=0, \quad i=0,1, \ldots, n_{2}-1 .
\end{aligned}
$$


TABLE 1. The approximate values of $\mu_{k}$ for $k=2,3,4$ for Example 6.1

\begin{tabular}{|c|c|}
\hline$k$ & $\mu_{k}$ \\
\hline 2 & 0.000813977 \\
3 & $9.440282 \times 10^{-6}$ \\
4 & $1.3411 \times 10^{-7}$ \\
\hline
\end{tabular}

Proof. For any given $\Delta>0$, we consider $u^{*} \in C^{n}[0,1]$ such that

$$
\tilde{J}\left[u^{*}\right]<\Delta \text {. }
$$

Such $u^{*}$ exists by the properties of minimum. On the other hand, $\tilde{J}$ is continuous on $C^{n}[0,1]$ so we have

$$
\left|\tilde{J}[u]-\tilde{J}\left[u^{*}\right]\right|<\Delta,
$$

provided that $\left\|u-u^{*}\right\|_{n}<\eta$. According to Weierstrass theorem given in [18], for large enough value of $k$, there exist $p_{k}(x) \in P_{k}[0,1]$ such that $\left\|p_{k}-u^{*}\right\|<\eta$. Moreover let $u_{k}$ be the element of $P_{k}[0,1]$ such that $\tilde{J}\left[u_{k}\right]=\hat{\mu}_{k}$, then using (5.7) and (5.8) we have

$$
0 \leq \tilde{J}\left[u_{k}\right] \leq \tilde{J}\left[p_{k}\right]<2 \Delta
$$

Since $\Delta>0$ is arbitrary, it follows that $\lim _{k \rightarrow \infty} \hat{\mu}_{k}=0$ and the result can be easily obtained.

\section{Test PROBlems}

Example 6.1. As the first example, we study the following singular multi-pantograph delay differential equation

$$
\begin{gathered}
u^{(2)}(x)+\frac{1}{x} u^{(1)}\left(\frac{x}{2}\right)+\frac{1}{x^{2}} u^{(1)}\left(\frac{x}{4}\right)-\frac{1}{x-1} u(x)=h(x), \quad 0<x \leqslant 1, \\
u(0)=1, \quad u(1)=e, \\
h(x)=e^{x}+\frac{1}{x} e^{\frac{x}{2}}+\frac{1}{x^{2}} e^{\frac{x}{4}}-\frac{1}{x-1} e^{x} .
\end{gathered}
$$

For above problem, we can see that exact solution is $u(x)=e^{x}$. Applying the method presented in Section 4, we achieve the approximate solution for the problem. The approximate values of $\mu_{k}$, for different number of basis functions $k$, are demonstrated in Table 1. Also Table 2 shows absolute error $\left|u_{k}(x)-u_{e x}(x)\right|$.

Example 6.2. In this example, we consider the following nonlinear equation

$$
\begin{aligned}
u^{(1)}(x)+2 u^{2}\left(\frac{x}{2}\right) & =1, \quad 0<x \leqslant 1, \\
u(0) & =0 .
\end{aligned}
$$

Noting to this problem, we can verify that the exact solution is $u(x) \sin (x)$. Table 3 shows the values of $\mu_{k}$ for different values of $k$ and Table 4 demonstrates the absolute error $\left|u_{k}(x)-u_{e x}(x)\right|$. 
TABLE 2. Absolute error for $k=2,3,4$ for Example 6.1

\begin{tabular}{|c|c|c|c|}
\hline$x$ & $k=2$ & $k=3$ & $k=4$ \\
\hline 0 & $1.71305 \times 10^{-7}$ & $2.2301 \times 10^{-8}$ & $5.19587 \times 10^{-9}$ \\
0.1 & 0.000846204 & $2.18047 \times 10^{-6}$ & $2.23933 \times 10^{-6}$ \\
0.2 & 0.0022476 & 0.00099343 & 0.0000524334 \\
0.3 & 0.00811796 & 0.00236922 & 0.0000851558 \\
0.4 & 0.0154792 & 0.00362989 & 0.0000788594 \\
0.5 & 0.0229106 & 0.00441537 & 0.0000430139 \\
0.6 & 0.0288417 & 0.004515 & $3.88571 \times 10^{-6}$ \\
0.7 & 0.0315373 & 0.00388327 & 0.0000111775 \\
0.8 & 0.0290793 & 0.00265722 & $8.8842 \times 10^{-6}$ \\
0.9 & 0.0193481 & 0.00117555 & 0.0000396475 \\
1 & $1.08041 \times 10^{-6}$ & $9.01796 \times 10^{-8}$ & $7.90223 \times 10^{-9}$ \\
\hline
\end{tabular}

TABLE 3. The approximate values of $\mu_{k}$ for $k=1,3,5$ for Example 6.2

\begin{tabular}{|c|c|}
\hline$k$ & $\mu_{k}$ \\
\hline 1 & 0.0124095 \\
3 & $2.19831 \times 10^{-6}$ \\
5 & $2.27916 \times 10^{-11}$ \\
\hline
\end{tabular}

TABLE 4. Absolute error for $k=1,3,5$ for Example 6.2

\begin{tabular}{|c|c|c|c|}
\hline $\mathrm{x}$ & $\mathrm{k}=1$ & $\mathrm{k}=3$ & $\mathrm{k}=5$ \\
\hline 0 & $3.37176 \times 10^{-6}$ & $1.27022 \times 10^{-9}$ & $1.89865 \times 10^{-14}$ \\
0.1 & 0.0142226 & 0.000169393 & $1.15815 \times 10^{-7}$ \\
0.2 & 0.0274443 & 0.000108566 & $4.02153 \times 10^{-7}$ \\
0.3 & 0.038681 & 0.0000550637 & $3.08125 \times 10^{-7}$ \\
0.4 & 0.0469649 & 0.000213911 & $2.1473 \times 10^{-7}$ \\
0.5 & 0.0513579 & 0.000289893 & $5.49804 \times 10^{-7}$ \\
0.6 & 0.0509607 & 0.000243804 & $3.47773 \times 10^{-7}$ \\
0.7 & 0.0449217 & 0.0000843014 & $1.61122 \times 10^{-7}$ \\
0.8 & 0.0324459 & 0.000123587 & $3.33028 \times 10^{-7}$ \\
0.9 & 0.0128025 & 0.00025052 & $1.60343 \times 10^{-7}$ \\
1 & 0.0146676 & 0.0000955398 & $4.00845 \times 10^{-8}$ \\
\hline
\end{tabular}

From the above numerical results, we can see that the proposed method is quite efficient.

\section{Conclusion}

This paper have developed an approximate method based on least squares, epsilon and Ritz methods for solving a general class of singular and nonsingular multipantograph equations. The convergence of the method has been extensively discussed 
and illustrative test examples have been included to demonstrate validity and applicability of the new method.

Acknowledgements. The authors are very much grateful to the editor and anonymous reviewers for their valuable comments and careful reading of the manuscript.

\section{REFERENCES}

[1] H. Brunner, Q. Haung and H. Xie, Discontinuous Galerkin method for delay differential equations of pantograph type, SIAM J. Numer. Anal. 48 (2010), 1944-1967.

[2] X. Chen and L. Wang, The Variational iteration method for solving a neutral functionaldifferential equation with proportional delays, Comput. Math. Appl. 59 (2010), 2696-2702.

[3] G. A. Derfal, Kato Problem for functional-differential equations and difference Schrodinger operators, Oper. Theory Adv. Appl. 46 (1990), 319-321.

[4] P. Du and F. Geng, A new method for solving singular multi-pantograph delay differential equation in reproducing kernel space, Appl. Math. Sci. 2 (2008), 1299-1305.

[5] P. A. Frickd and J. Stech, Epsilon-ritz method for solving optimal control problems: Useful parallel solution method, J. Optim. Theory Appl. 79 (1993), 31-58.

[6] P. Hu, C. Huang and S. Wu, Asymptotic stability of linear multistep methods for nonlinear neutral delay differential equations, Appl. Math. Comput. 211 (2009), 95-101.

[7] E. Ishiwata and Y. Muroya, Rational approximation method for delay differential equations with proportional delay, Appl. Math. Comput. 187 (2007), 741-747.

[8] S. Javadi, E. Babolian and Z. Taheri, Solving generalized pantograph equations by shifted orthonormal Bernstein polynomials, J. Comput. Appl. Math. 303 (2016), 1-14.

[9] D. Li and M. Z. Liu, Runge-Kutta methods for the multi-pantograph delay equation, Appl. Math. Comput. 163 (2005), 383-395.

[10] M. Z. Liu and Z. W. Yang, The stability of modified runge-kutta methods for the pantograph equation, Math. Comp. 75 (2006), 1201-1215.

[11] M. Z. Liu and D. Li, Properties of analytic solution and numerical solution of multi-pantograph equation, Appl. Math. Comput. 155 (2004), 853-871.

[12] A. Lotfi and S. A. Yousefi, Epsilon-Ritz method for solving a class of fractional constrained optimization problems, J. Optim. Theory Appl. 163 (2014), 884-899.

[13] W. Lv and H. Li, Numerical stability of higher-order derivative methods for the pantograph equation, Appl. Math. Comput. 218 (2012), 5739-5775.

[14] K. Mahler, On a special functional equation, J. Lond. Math. Soc. 15 (1940), 115-123.

[15] Y. Muroya, E. Ishivata and H. Brunner, On the attainable order of collocation methods for pantograph integro-differential equations, J. Comput. Appl. Math. 152 (2003), 347-366.

[16] J. R. Ockendon and A. B. Tayler, The dynamics of a current collection system for an electric locomotive, Proc. Roy. Soc. Edinburgh Sect. A 322 (1971), 447-468.

[17] P. Rahimkhani, Y. Ordokhani and E. Babolian, Numerical solution of fractional pantograph differential equations by using generalized fractional-order Bernoulli wavelet, J. Comput. Appl. Math. 309 (2017), 493-510.

[18] H. L. Royden, Real Analysis, 3rd ed. Macmillan Publishing Company U.S.A, 1988.

[19] A. Saadatmandi and M. Dehghan, Variational iteration method for solving a generalized pantograph equation, Comput. Math. Appl. 58 (2009), 2190-2196.

[20] S. Sedaghat, Y. Ordokhani and M. Dehghan, Numerical solution of the delay differential equations of pantograph type via Chebyshev polynomials, Commun. Nonlinear Sci. Numer. Simul. 17 (2012), 4815-4830. 
[21] M. Sezer, S. Yalcinbas and N. Sahin, Approximate solution of multi-pantograph equation with variable coefficients, J. Comput. Appl. Math. 214 (2008), 406-416.

[22] M. Sezer, S. Yalcinbas and M. Gulsu, A Taylor polynomial approach for solving generalized pantograph equations with nonhomogeneus term, Int. J. Comput. Math. 85 (2008), 1055-1063.

[23] M. Sezer and A. Akyus-Dascioglu, A Taylor method for numerical solution of generalized pantograph equations with linear functional argument, J. Comput. Appl. Math. 200 (2007), 217225.

[24] E. Sokhanvar and S. A. Yousefi, An effective method for numerical solution of delay differential equations of multi-pantograph type, Journal of Advanced Research in Dynamical and Control Systems, 5 (2013), 20-36.

[25] W. Wang and S. Li, On the one-leg $\theta$-method for solving nonlinear neutral functional differential equations, Appl. Math. Comput. 193 (2007), 285-301.

[26] W. Wang, T. Qin and S. Li, Stability of one-leg $\theta$-method for nonlinear neutral differential equations with proportional delay, Appl. Math. Comput. 213 (2009), 177-183.

[27] Z. Yu, Variational iteration method for solving the multi-pantograph delay equation, Phys. Lett. A 372 (2008), 6475-6479.

[28] E. Yusufoglu, An efficient algorithm for solving generalized pantograph equations with linear functional argument, Appl. Math. Comput. 217 (2010), 3591-3595.

[29] S. Yuzbasi, N. Sahin and M. Sezer, A Bessel collocation method for numerical solution of generalized pantograph equations, Numer. Methods Partial Differential Equations, 28 (2012), 11051123.

[30] S. Yuzbasi, An efficient algorithm for solving multi-pantograph equation systems, Comput. Math. Appl. 64 (2012), 589-603.

[31] S. Yuzbasi, M. Aynigul and M. Sezer, A collocation method using Hermite polynomials for approximate solution of pantograph equation, J. Franklin Inst. 348 (2011), 1128-1139.

[32] S. Yuzbasi and M. Sezer, Shifted Legendre approximation with the residual correction to solve pantograph-delay type differential equations, Appl. Math. Model. 39 (2015), 6529-6542.

\footnotetext{
${ }^{1}$ Department of Mathematics, Faculty of Mathematical Sciences, Shahid Beheshti University, General Campus, Evin, Tehran 19839, Iran

E-mail address: s-yousefi@sbu.ac.ir
} 